\title{
Fast Food and Neighborhood Stroke Risk
}

\author{
Lewis B. Morgenstern, MD, ${ }^{1,2}$ James D. Escobar, MPH, ${ }^{2}$ Brisa N. Sánchez, PhD, ${ }^{3}$ Rebecca Hughes, BA, ${ }^{1}$ \\ Belinda G. Zuniga, CNA, ${ }^{2}$ Nelda Garcia, BS, ${ }^{1}$ and Lynda D. Lisabeth, $\mathrm{PhD}^{1,2}$
}

Objective: To investigate the association between the number of fast food restaurants and ischemic stroke in neighborhoods. Methods: This work was a prespecified part of the Brain Attack in Corpus Christi (BASIC) project. Ischemic stroke cases were prospectively ascertained in Nueces County, Texas. Home addresses were geocoded and used to establish the census tract for each stroke case. Census tracts were used as proxies for neighborhoods $(n=64)$. Using a standard definition, fast food restaurants were identified from a commercial list. Poisson regression was used to study the association between the number of fast food restaurants in the neighborhood, using a 1-mile buffer around each census tract, and the risk of stroke in the neighborhood. Models were adjusted for demographics and neighborhood socioeconomic status (SES).

Results: There were 1,247 completed ischemic strokes from January 2000 through June 2003 and 262 fast food restaurants. The median number of fast food restaurants per census tract including buffer was 22 (interquartile range, 12-33). Adjusting for neighborhood demographics and SES, the association of fast food restaurants with stroke was significant $(p=0.02)$. The association suggested that the risk of stroke in a neighborhood increased by $1 \%$ for every fast food restaurant (relative risk, 1.01; $95 \%$ confidence interval $[\mathrm{CI}], 1.00-1.01)$. The relative risk of stroke comparing neighborhoods in the 75 th to the 25 th percentile of the distribution of fast food restaurants was 1.13 (95\% CI, 1.02-1.25).

Interpretation: Controlling for demographic and SES factors, there was a significant association between fast food restaurants and stroke risk in neighborhoods in this community-based study.

Ann Neurol 2009;66:165-170

By affecting wide ranging behaviors and sociocognitive dimensions, such as diet, exercise, stress, and depression, many aspects of neighborhood residence may influence health, including available recreational space, crime, public transportation, and availability of food stores. The contextual effects of a neighborhood on disease risk have received close attention, ${ }^{1}$ but very little with respect to stroke. ${ }^{2}$ Stroke is a disease of atherosclerosis. Risk factors include hypertension, obesity, and hyperlipidemia. ${ }^{3}$ Fast food options have become a quickly growing and universal phenomenon. ${ }^{4}$ These establishments offer a quick and inexpensive meal. In general, many offerings are also high in fat and salt, and rarely provide fruit, vegetables, or whole grains. Fast food restaurants tend to cluster in neighborhoods that are more economically disadvantaged, ${ }^{5,6}$ and in areas with high proportions of minority residents. ${ }^{7,8}$ Neighborhood disadvantage has been linked to stroke risk. $^{2}$

Accessibility to fast food restaurants may be one pathway by which neighborhood disadvantage contributes to atherosclerosis. ${ }^{7}$ Another possibility is that neighborhoods that have high fast food restaurant densities have less options for healthy eating. ${ }^{9}$ Regardless of the mechanism for increased disease risk, if fast food restaurant density is associated with stroke risk, then appropriate public health interventions in specific neighborhoods can be suggested.

Other risk factors that go beyond traditional biologic and social risk factors may potentially contribute risk for common, severe diseases such as stroke. We studied the association of the density of fast food restaurants with ischemic stroke in neighborhoods as part of a population-based study in South Texas.

\section{Materials and Methods}

This study took place in Corpus Christi, Nueces County, Texas as a preplanned analysis of the Brain Attack Surveillance in Corpus Christi (BASIC) project. The methods of the BASIC project were previously reported. ${ }^{10,11}$ Briefly, all cerebrovascular disease cases among those $\geq 45$ years old were prospectively ascertained from all hospitals, neurologist offices, and the county coroner from January 2000 through June 2003. Completed ischemic stroke cases were validated from source documentation by board eligible/certified neurologists blinded to subject age, ethnicity, and residence. Cases of transient ischemic attack, intracerebral hemorrhage, and subarachnoid hemorrhage were excluded. Stroke case definitions were based on published international clinical cri-
From the ${ }^{1}$ Stroke Program, University of Michigan Medical School, ${ }^{2}$ Department of Epidemiology, University of Michigan School of Public Health, and ${ }^{3}$ Department of Biostatistics, University of Michigan School of Public Health, Ann Arbor, MI.

Address correspondence to Dr Morgenstern, University of Michigan Cardiovascular Center, Room 3194, 1500 East Medical Center
Dr. SPC \#5855, Ann Arbor, MI 48109-5855. E-mail: LMorgens@umich.edu

Potential conflict of interest: Nothing to report.

Received Dec 16, 2008, and in revised form Feb 16, 2009. Accepted for publication Apr 3, 2009. Published online in Wiley InterScience (www.interscience.wiley.com). DOI: 10.1002/ana.21726 
teria. ${ }^{12}$ Corpus Christi makes up $>95 \%$ of the county's population, and is an urban environment with mass transport facilities (buses). The county population is approximately 300,000 residents, and the location is on the Texas Gulf Coast. It is a nonimmigrant community, with long-term residence and little influx or efflux of individuals. ${ }^{13}$ Corpus Christi is about 200 miles from Houston and San Antonio, and the surrounding counties are sparsely populated, allowing for complete case capture. This study was approved by the University of Michigan institutional review board (IRB) and by the IRBs of the 2 hospital systems in Corpus Christi.

United States census tracts ${ }^{14}$ were used as proxies for neighborhoods ( $\mathrm{n}=64)$. Home addresses were geocoded, and used to identify the census tract for each stroke case. Demographics, stroke risk factors, and insurance status were collected from the medical record.

Fast food restaurants were identified from a commercial list (Reference USA, US Business Database), and defined as having $\geq 2$ of the following characteristics: expedited food service, takeout business, limited or no wait staff, or customers who pay prior to receiving food. Each fast food restaurant was geocoded, and a count of the number of fast food restaurants per census tract was generated. To create a more realistic "dining area," a 1-mile buffer around each census tract was created, and restaurants falling within the buffered area and within the county were counted for the given census tract. Use of similar distance buffers is a previously used methodology. ${ }^{15}$ Distances between stroke cases and fast food restaurants were calculated by first calculating the difference in degrees longitude and degrees latitude between the 2 locations. The differences in degrees were then converted to miles. The Pythagorean theorem was then used to calculate the distance. Of the fast food restaurants identified, 75\% were in business in 2003 and 50\% in 1994, attesting to their longevity in the neighborhoods.

US Census data from $2000^{14}$ was the source for census tract-level population counts and neighborhood variables (median household income; median value of occupied housing units; percent of households receiving interest, dividend, or net rental income; percent of adults who complete high school; percent of adults who complete college; and percent of adults whose occupation is classified as managerial or professional). A summary score representing neighborhood socioeconomic status was constructed from these 6 variables based on previously published methods. ${ }^{16}$ The summary score was constructed as follows. For each of the 6 census variables, a mean and standard deviation were calculated across census tracts. For each census tract, a $Z$ score for each census variable was then estimated by subtracting the mean and dividing by the standard deviation for that variable. The $Z$ scores for each variable were then summed to get a neighborhood score for the given census tract, with increasing score representing increasing neighborhood advantage. The median and interquartile ranges (IQR) for each element of the summary score and for the overall summary score for the population were previously reported. ${ }^{2}$

\section{Statistical Analysis}

Median number of fast food restaurants per census tract and median distance of the ischemic stroke cases' homes to a fast food restaurant were calculated. Stroke rates per 1,000 population were calculated for each census tract. Using ArcGIS software, the quintiles of the distribution of stroke rates were calculated. Poisson regression was used to study the association between the number of fast food restaurants in the neighborhood (modeled continuously) and the risk of stroke events in the neighborhood. That is, the stroke counts were the outcome for the regression, and population size of the census tract was used as an offset. Robust standard errors were used to compute confidence intervals for the effect estimates. Models were run unadjusted, adjusted for demographics (age, gender, race/ethnicity), and adjusted for demographics and neighborhood socioeconomic status. Age was modeled categorically as dummy variables representing ages 45-59 years (referent), 60-74 years, and $\geq 75$ years. Gender and race/ethnicity were modeled as dummy variables with men and non-Hispanic whites (NHWs) as the referents, respectively. Neighborhood score was modeled continuously. Models were also run stratified by age group, gender, and race/ethnicity to determine if the association of the number of fast food restaurants and the count of stroke events in the neighborhood differed across levels of these variables. Effect modification between the demographic variables and the number of fast food restaurants was formally tested, as was effect modification between neighborhood score and the number of fast food restaurants.

In secondary analyses, logistic regression was used to study the associations between the number of fast food restaurants in the neighborhood and stroke risk factors among the stroke cases. To account for the correlation among cases living in the same neighborhood, the models were estimated using generalized estimating equations with an exchangeable correlation structure. ${ }^{17}$ Models were run unadjusted and adjusted for demographics (age, gender, race/ethnicity) and neighborhood socioeconomic status. Gender and race/ethnicity were modeled as dummy variables as described above. Age and neighborhood score were modeled continuously.

\section{Results}

There were 2,984 validated cerebrovascular events during the study period. Of the 2,984, 2,727 were geocoded to the census tract level. The remaining $9.1 \%$ were only able to be geocoded to the zip code level and were excluded. Of the 2,727, 1,581 were ischemic strokes. Only NHWs and Mexican Americans (MAs) were included in this study due to small numbers for other race-ethnic groups, resulting in 1,468 events. For those individuals with multiple ischemic strokes, only the first ischemic stroke event was considered. Therefore, the final population for analysis was limited to 1 stroke per individual, for a total of 1,336 stroke cases. Of the 1,336 cases, 13 were nonresidents of Nueces County, and were excluded. Because we were interested in measuring exposure to fast food restaurants over the time period relevant to development of stroke, cases who were currently residing in a nursing home or assisted living facility $(\mathrm{n}=76)$ were also excluded. This left 1,247 stroke cases for the final analysis.

Table 1 provides the subjects' demographic and clin- 
ical characteristics. There were 262 fast food restaurants in the county. The median number of fast food restaurants per census tract including the 1-mile buffer was 22 (IQR, 12-33). The median distance of the ischemic stroke cases' homes to a fast food restaurant was 0.32 miles (IQR, 0.20-0.50).

The Figure shows the distribution of fast food restaurants in the community superimposed on the stroke rates in each neighborhood. Table 2 provides the data on the association of fast food restaurants and ischemic stroke. Adjusting for demographics and neighborhood score, the association of the number of fast food restaurants with stroke was significant $(p=0.02)$. The association suggests that the risk of stroke in a neighborhood increased by $1 \%$ for every fast food restaurant (relative risk, 1.01; 95\% confidence interval [CI], $1.00-1.01)$ in the neighborhood. The relative risk of stroke comparing neighborhoods in the 75th to the 25th percentile of the distribution of fast food restaurants in this community was $1.13 \quad(95 \% \mathrm{CI}$, 1.02-1.25). Age demonstrated a significant interaction with fast food restaurants and ischemic stroke risk, such that the association is present in the younger age categories but not in the oldest age category (Table 3). Although the interaction between ethnicity and fast food on the risk of stroke was not significant, the association of fast food with stroke was only present for MAs (Table 3). Gender did not demonstrate a significant interaction with fast food and the risk of ischemic

Table 1. Demographic and Clinical Characteristics of Study Subjects, Corpus Christi, Nueces County, Texas, January 2000-June 2003, $N=1,247$

No. $(\%)$

Age (mean, SD)

Women

MA

Hypertension

Diabetes

High cholesterol

Current smoker

History stroke/TIA

Coronary artery disease

Atrial fibrillation

Alcohol $>2$ per day

$73(6)$

Any medical insurance $1,175(94)$

Four cases were missing data on all clinical characteristics, 1 case was missing data on coronary artery disease, 4 cases were missing data on alcohol, 59 cases were missing data on smoking, and 2 cases were missing data on insurance. SD = standard deviation; $\mathrm{MA}=$ Mexican American; TIA $=$ transient ischemic attack.

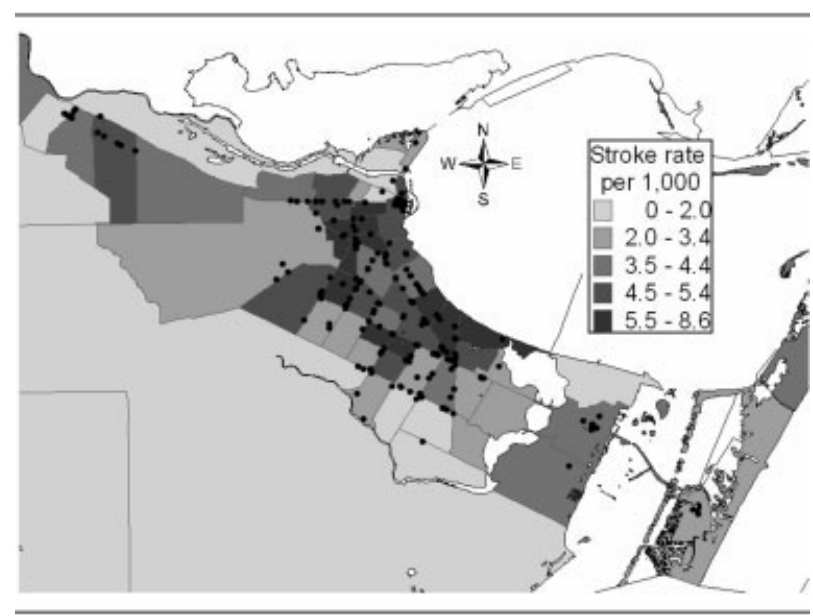

Fig. Quintiles of stroke rates and distribution of fast food restaurants (identified by black dots) in Corpus Christi, Nueces County, Texas, January 2000-June 2003.

stroke. There was a borderline significant interaction between neighborhood score and fast food restaurants, suggesting the association of fast food with stroke is greater in neighborhoods with higher socioeconomic status (SES).

Table 4 displays the associations of stroke risk factors with the number of fast food restaurants in neighborhoods among stroke cases. Only atrial fibrillation demonstrated a significant association. Atrial fibrillation was found in $16.0 \%$ of NHWs and $8.6 \%$ of MA stroke cases.

\section{Discussion}

This study demonstrates an association of the density of fast food restaurants and ischemic stroke cases in neighborhoods of a community. Although the strength of the association is not large, the prevalence of fast food restaurants in many communities throughout the world suggests a large potential public health risk. Given the study design, however, it is unknown whether this finding is due to increased consumption of fast food among stroke cases or other individual or neighborhood factors. Future work should include investigating the independent effect of fast food density on stroke risk after adjustment for individual-level risk factors in a multilevel fashion. Recent studies have identified neighborhood factors that serve as independent cardiovascular risk factors over and above individual risk factors. ${ }^{18}$

Stroke risk factors that may be linked to fast food consumption include hypertension, obesity, diabetes and hyperlipidemia. Consumption of fast food is associated with increased salt and caloric intake, and suggests a trend toward weight gain and obesity. ${ }^{8,19}$ A clustering of fast food restaurants with mortality and acute coronary syndromes has been noted in Ontario, Canada, and the authors suggest targeting these high-risk communities 


$\begin{aligned} & \text { Table 2. Unadjusted and Multivariate Adjusted Associations of Fast Food Restaurants and Ischemic Stroke } \\ & \begin{array}{l}\text { Model 1, RR } \\ (\mathbf{9 5 \%} \mathbf{C I})\end{array}\end{aligned}$
$\begin{aligned} & \text { Model 2, RR } \\ & (\mathbf{9 5 \%} \mathbf{C I})\end{aligned}$

with vascular disease prevention interventions. ${ }^{20}$ Simply posting calorie information at fast food restaurants is associated with healthier food choices. ${ }^{21}$ The close proximity of fast food restaurants to schools ${ }^{22}$ and the frequent use by adolescents has been implicated in the epidemic of childhood obesity. ${ }^{8}$ It also suggests that interventions aimed at reducing fast food consumption should include children in the educational target group. Children have been a successful target for stroke educational interventions in the past. ${ }^{23,24}$ Researchers have shown that the relative low cost of fast food compared with fruit and vegetables drives consumption. Lower fruit and vegetable costs and/or higher fast food prices may promote healthy eating, ${ }^{25}$ and serves as a possible target for intervention.

In stratified models, we studied whether the relationship of fast food restaurants and ischemic stroke differed among subgroups of the population based on ethnicity and age. Significant associations between fast food restaurants and stroke were noted for those of younger (45-59 and 60-74 years) but not older ages. Fast food restaurants may be 1 pathway that explains the association of neighborhood and stroke risk in those $<75$ years of age. ${ }^{2}$ Selective survival, the phenomena whereby the cohort that survives to older ages may have different risk factor vulnerabilities, may explain in part why the association of fast food and stroke risk was only noted in those $<75$ years of age. ${ }^{26}$ Also, additional information about the relative consumption of fast food over the lifespan is needed and may shed light on the lack of an association in the oldest age group.

In the current study, the association of fast food restaurants with stroke risk was greater in neighborhoods with higher neighborhood score (Table 3). Previous work in other diseases has suggested that more disadvantaged neighborhoods were associated with a high density of fast food restaurants and disease risk. ${ }^{7}$ We have previously shown that the association of SES and stroke risk in this community is complicated and dependent on interactions of age and gender. ${ }^{2}$

Another interesting finding was that the association of fast food restaurants and stroke was seen in MAs, not NHWs. There are no reports comparing fast food consumption in MAs and NHWs. The reason residents of more advantaged neighborhoods and MAs have stronger associations of fast food and stroke risk is uncertain but may be due to complex interactions of age, gender, neighborhood score, and ethnicity of the residents of these neighborhoods.

In a secondary analysis, we found that risk factors among stroke cases such as hypertension, diabetes, and hyperlipidemia were not associated with the number of fast food restaurants in neighborhoods. Only atrial fibrillation, which is found in a small number of stroke cases, was associated with fast food restaurants. Further, atrial fibrillation is significantly less common in MAs, the ethnic group for which the fast food-stroke association seems present. This secondary analysis requires caution. Because risk factor data were not available from the gen- 
eral population, we could only examine stroke risk factors in cases. If the association of fast food with stroke risk is not mediated by conventional risk factors, perhaps the density of fast food restaurants serves as a surrogate marker for other, novel neighborhood components that confer stroke risk. For example, air pollution has been associated with stroke risk, ${ }^{27}$ and pollutant sources may cluster with fast food restaurants.

This study has limitations. Inherent in any ecological design is the inability to adjust associations based on individual risk factors, although we were able to adjust for age, gender, race/ethnicity, and neighborhood disadvantage given the availability of this information in the Census. We do not know whether the stroke cases actually consumed fast food or just resided in neighborhoods with higher numbers of fast food restaurants. We did not consider other food establishments. Further, we classified fast food restaurants based on standard criteria and used a commercial list to identify establishments; however, we cannot be certain that some fast food restaurants were not misclassified or omitted.

Table 3. Association of Fast Food Restaurants and Ischemic Stroke Stratified by Ethnicity, Gender, Age, and Neighborhood SES

\begin{tabular}{|c|c|c|}
\hline & $\operatorname{RR}(95 \% \mathrm{CI})^{\mathbf{a}}$ & $p_{\text {interaction }}{ }^{b}$ \\
\hline \multicolumn{3}{|l|}{ Ethnicity } \\
\hline Non-Hispanic White & $1.03(0.95-1.11)$ & \multirow[t]{2}{*}{0.18} \\
\hline Mexican American & $1.10(1.01-1.18)$ & \\
\hline \multicolumn{3}{|l|}{ Gender } \\
\hline Male & $1.05(1.00-1.11)$ & \multirow[t]{2}{*}{0.61} \\
\hline Female & $1.07(1.01-1.13)$ & \\
\hline \multicolumn{3}{|l|}{ Age, yr } \\
\hline $45-59$ & $1.09(1.03-1.16)$ & \multirow[t]{3}{*}{0.05} \\
\hline $60-74$ & $1.10(1.01-1.20)$ & \\
\hline $75+$ & $1.00(0.92-1.08)$ & \\
\hline \multicolumn{3}{|l|}{ Neighborhood SES ${ }^{c}$} \\
\hline High & $1.21(1.06-1.38)$ & \multirow[t]{2}{*}{0.07} \\
\hline Low & $1.02(0.89-1.18)$ & \\
\hline \multicolumn{3}{|c|}{ 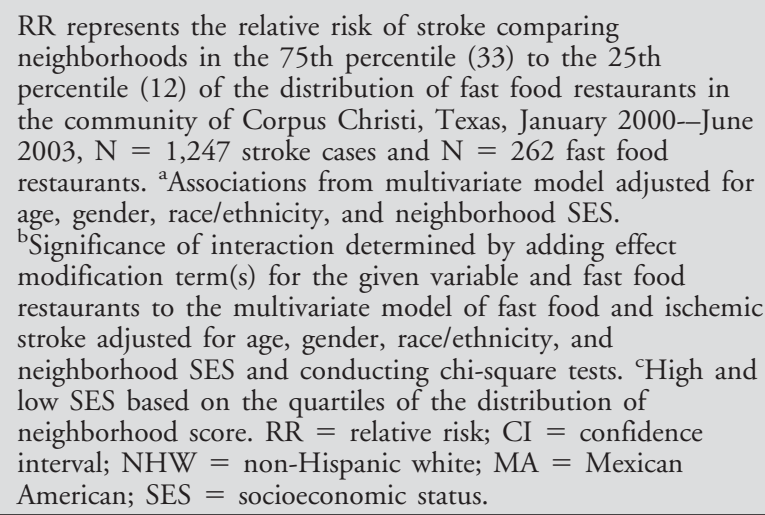 } \\
\hline
\end{tabular}

Table 4. Unadjusted and Multivariate Adjusted Associations of Fast Food Restaurants and Stroke Risk Factors

\begin{tabular}{|c|c|c|}
\hline & $\begin{array}{l}\text { Model 1, RR } \\
\quad(95 \% \text { CI })\end{array}$ & $\begin{array}{l}\text { Model 2, RR } \\
(95 \% \text { CI })\end{array}$ \\
\hline Hypertension & $0.99(0.83-1.18)$ & $0.98(0.82-1.16)$ \\
\hline Diabetes & $1.08(0.82-1.41)$ & $0.98(0.79-1.22)$ \\
\hline High cholesterol & $0.97(0.73-1.30)$ & $0.99(0.74-1.32)$ \\
\hline Current smoker & $0.83(0.64-1.08)$ & $0.87(0.68-1.12)$ \\
\hline $\begin{array}{l}\text { History stroke/ } \\
\text { TIA }\end{array}$ & $0.93(0.81-1.08)$ & $0.90(0.81-1.31)$ \\
\hline $\begin{array}{l}\text { Coronary artery } \\
\text { disease }\end{array}$ & $1.03(0.81-1.31)$ & $1.03(0.81-1.31)$ \\
\hline Atrial fibrillation & $1.31(1.08-1.59)$ & $1.25(1.03-1.52)$ \\
\hline \multicolumn{3}{|c|}{$\begin{array}{l}\text { Data represent the RR of stroke comparing neighborhoods in } \\
\text { the } 75 \text { th percentile ( } 33) \text { to the } 25 \text { th percentile }(12) \text { of the } \\
\text { number of fast food restaurants in the community of Corpus } \\
\text { Christi, Texas, January } 2000 \text {-June } 2003, N=1,247 \text { stroke } \\
\text { cases and } N=262 \text { fast food restaurants. Model } 1= \\
\text { unadjusted; Model } 2=\text { adjusted for age, gender, race/ethnicity } \\
\text { and neighborhood socioeconomic status. RR = relative risk; } \\
\mathrm{CI}=\text { confidence interval; TIA = transient ischemic attack. }\end{array}$} \\
\hline
\end{tabular}

In summary, this study demonstrated an association of the number of fast food restaurants with risk of stroke, especially among MAs, those $<75$ years old, and those of higher SES, in neighborhoods of a South Texas community. Further research is needed to determine if this association is due to increased fast food consumption in these neighborhoods, other neighborhood factors that place residents at risk, or differences in individual risk factor profiles. This finding has a large potential public health impact due to the high prevalence and worldwide spread of fast food restaurants and the enormous public health burden of stroke.

This study was funded by National Institutes of Health R01 NS38916.

\section{References}

1. Sampson RJ. The neighborhood context of well-being. Perspect Biol Med 2003;46:S53-S64.

2. Lisabeth LD, Diez Roux AV, Escobar JD, Smith MA, Morgenstern LB. Neighborhood environment and risk of ischemic stroke: the Brain Attack Surveillance in Corpus Christi (BASIC) project. Am J Epidemiol 2007;165:279-287.

3. Goldstein LB, Adams R, Alberts MJ, et al. Primary prevention of ischemic stroke: a guideline from the American Heart Association/American Stroke Association stroke council. Cosponsored by the Atherosclerotic Peripheral Vascular Disease Interdisciplinary Working Group; Cardiovascular Nursing Council; Clinical Cardiology Council; Nutrition, Physical Activity, and Metabolism Council; and the Quality of Care and Outcomes Research Interdisciplinary Working Group. Circulation 2006;113:e873-e923. 
4. Wang MC, Cubbin C, Ahn D, Winkleby MA. Changes in neighbourhood food store environment, food behaviour and body mass index, 1981-1990. Public Health Nutr 2008;11: 963-970.

5. Hemphill E, Raine K, Spence JC, Smoyer-Tomic KE. Exploring obesogenic food environments in Edmonton, Canada: the association between socioeconomic factors and fast-food outlet access. Am J Health Promot 2008;22:426-432.

6. Macdonald L, Cummins S, Macintyre S. Neighbourhood fast food environment and area deprivation-substitution or concentration? Appetite 2007;49:251-254.

7. Kwate NO, Yau CY, Loh JM, Williams D. Inequality in obesigenic environments: Fast food density in New York City. Health Place 2009;15:364-373.

8. Schmidt M, Affenito SG, Striegel-Moore R, et al. Fast-food intake and diet quality in black and white girls: the National Heart, Lung, and Blood Institute Growth and Health study. Arch Pediatr Adolesc Med 2005;159:626-631.

9. Moore LV, Diez Roux AV. Associations of neighborhood characteristics with the location and type of food stores. Am J Public Health 2006;96:325-331.

10. Morgenstern LB, Smith MA, Lisabeth LD, et al. Excess stroke in Mexican Americans compared with non-Hispanic whites: the Brain Attack Surveillance in Corpus Christi project. Am J Epidemiol 2004;160:376-383.

11. Smith MA, Risser JM, Moye LA, et al. Designing multi-ethnic stroke studies: the Brain Attack Surveillance in Corpus Christi (BASIC) project. Ethn Dis 2004;14:520-526.

12. Gillum RF, Fortmann SP, Prineas RJ, Kottke TE. International diagnostic criteria for acute myocardial infarction and acute stroke. Am Heart J 1984;108:150-158.

13. Morgenstern LB, Steffen-Batey L, Smith MA, Moye LA. Barriers to acute stroke therapy and stroke prevention in Mexican Americans. Stroke 2001;32:1360-1364.

14. United States Census 2000. www.uscensus.gov. Accessed February $2,2009$.

15. Crawford DA, Timperio AF, Salmon JA, et al. Neighbourhood fast food outlets and obesity in children and adults: the CLAN study. Int J Pediatr Obes. 2008;3:249-256.
16. Diez-Roux AV, Nieto FJ, Muntaner C, et al. Neighborhood environments and coronary heart disease: a multilevel analysis. Am J Epidemiol 1997;146:48-63.

17. Zeger SL, Liang KY. An overview of methods for the analysis of longitudinal data. Stat Med. 1992;11:1825-1839.

18. Augustin T, Glass TA, James BD, Schwartz BS. Neighborhood psychosocial hazards and cardiovascular disease: the Baltimore Memory Study. Am J Public Health 2008;98:1664-1670.

19. Rosenheck R. Fast food consumption and increased caloric intake: a systematic review of a trajectory towards weight gain and obesity risk. Obes Rev 2008;9:535-547.

20. Alter DA, Eny K. The relationship between the supply of fastfood chains and cardiovascular outcomes. Can J Public Health 2005;96:173-177.

21. Bassett MT, Dumanovsky T, Huang C, et al. Purchasing behavior and calorie information at fast-food chains in New York City, 2007. Am J Public Health 2008;98:1457-1459.

22. Simon PA, Kwan D, Angelescu A, Shih M, Fielding JE. Proximity of fast food restaurants to schools: do neighborhood income and type of school matter? Prev Med. 2008;47: $284-288$.

23. Williams O, Noble JM. 'Hip-hop' stroke: a stroke educational program for elementary school children living in a high-risk community. Stroke 2008;39:2809-2816.

24. Morgenstern LB, Gonzales NR, Maddox KE, et al. A randomized, controlled trial to teach middle school children to recognize stroke and call 911: the Kids Identifying and Defeating Stroke project. Stroke 2007;38:2972-2978.

25. Beydoun MA, Powell LM, Wang Y. The association of fast food, fruit and vegetable prices with dietary intakes among us adults: is there modification by family income? Soc Sci Med 2008;66:2218-2229.

26. Markides KS, Machalek R. Selective survival, aging and society. Arch Gerontol Geriatr 1984;3:207-222.

27. Lisabeth LD, Escobar JD, Dvonch JT, et al. Ambient air pollution and risk for ischemic stroke and transient ischemic attack. Ann Neurol 2008;64:53-59. 